LETTER TO THE EDITOR

\title{
Decline in circulating neuropeptide $Y$ levels in normal elderly human subjects
}

\author{
P Chiodera, R Volpi, S Pilla, S Cataldo and V Coiro \\ Department of Internal Medicine and Biomedical Sciences, School of Medicine, University of Parma, Parma, Italy \\ (Correspondence should be addressed to P Chiodera; Fax: +39-0521-991490)
}

Normal aging in humans and rats is associated with a decline in food intake and body weight (BW) (1). This condition is defined as 'anorexia of aging' and is considered a physiological phenomenon of normal aging; nevertheless this anorexia is often responsible for undernutrition and consequent physical deterioration (2). In fact, long-term tissue catabolic processes significantly reduce not only body fat, but also lean body mass, leading to osteoporosis, sarcopenia, impaired immunity and parenchymatous organ failure. These pathological events worsen quality of life and shorten life span (1).

A variety of studies indicate that age-related anorexia is associated with alterations in the homeostatic mechanisms controlling hunger and satiety in the central nervous system. An important role in the pathogenesis of anorexia of aging has been attributed to age-related changes in the leptin-NPY (neuropeptide Y) feeding pathway. Particularly, reduction in function and content of NPY in several cerebral areas has been demonstrated during advanced ages in rats (for review see 1). These demonstrations required the use of invasive methods, and thus could not be confirmed in the human species. In recent years, it has become possible to measure plasma circulating levels of NPY and leptin in humans. Therefore, we decided to measure these neurochemicals in the blood of senescent human subjects to verify whether significant changes in plasma NPY and/or leptin concentrations occur with aging and whether these alterations correlate with other parameters, such as age and body mass index (BMI). For this purpose, 36 normal men aged 23-82 years participated in this study after giving informed consent. Subjects were divided into three age groups (group I: $23-38$ years, mean \pm s.E. $30.2 \pm 2.7(n=12)$; group II: $40-58$ years, mean $51.7 \pm 2.4(n=11)$; group III: $63-82$ years, mean $73.7 \pm 1.7(n=13))$. Men were in good physical health, fully ambulatory and well nourished. Men of similar body constitution were chosen (group I: BW $68.4 \pm 6.9 \mathrm{~kg}$ (mean \pm s.E.), mean BMI 22.3; group II: BW $69.0 \pm 7.1 \mathrm{~kg}$, BMI 22.4; group III: BW $68.0 \pm$ $7.3 \mathrm{~kg}$, BMI 22.1). None of the subjects had clinical features or laboratory evidence of endocrine, metabolic or other intercurrent disease. Subjects fasted from at least midnight and blood samples were taken at $0830 \mathrm{~h}$. Serum leptin and plasma NPY were measured by RIA, using commercial kits. The intra- and interassay coefficients of variation were 5 and $3.2 \%$, and 8 and $11.6 \%$ respectively. The sensitivity of the method was $0.1 \mathrm{ng} / \mathrm{ml}$ for leptin and $6 \mathrm{pmol} / \mathrm{l}$ for NPY. Statistical analysis was performed with an unpaired $t$ test and a linear correlation coefficient, as appropriate. Values are reported as means \pm s.E.

Whereas plasma leptin levels were similar in all age groups, a significant decrease of NPY concentration
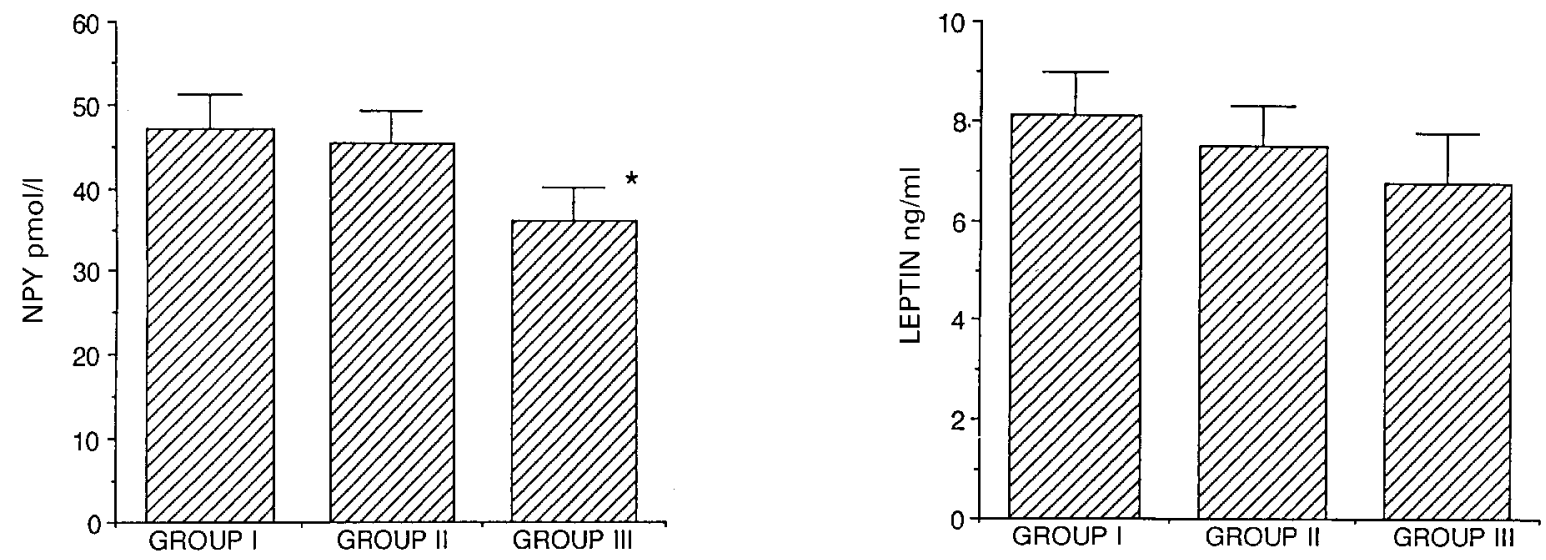

Figure 1 NPY and leptin levels in subjects of different ages. ${ }^{*} P<0.05$ for group III vs group I and group II. 
was observed in the elderly subjects (group III vs group I, $P<0.05$; group III vs group II, $P<0.05$ ) (Fig. 1). When plasma NPY levels were combined with BMI values, no significant correlation was observed in any group. Similarly, NPY values did not correlate with age in group I and II. In contrast a significant correlation was observed between plasma NPY concentration and age in group III $(r=0.84 ; P<0.001)$.

Thus, in agreement with studies reporting a decrease in NPY concentrations in the rat hypothalamus, frontal cortex, hippocampus and paraventricular nucleus, we have observed a decline in plasma NPY levels in humans that significantly correlated with increasing age. Observations in both rats and humans suggest an age-related decline in NPY activity. The role of NPY in the control of the feeding system consists of the stimulation of ways that favor food intake and energy storage (3). A reduction in NPY feeding-stimulatory actions near the end of life makes NPY failure a likely candidate effector in the anorexia of aging. In contrast, leptin is secreted from adipose tissue and inhibits feeding probably through inhibition of NPY gene transcription. Our observation of normal circulating levels of leptin in the older group without any correlation with NPY, BMI and age argues against the possibility that increased leptin activity at the site of feeding-control structures affects the anorectic elderly subjects.

\section{References}

1 Blanton CA, Horwitz B \& McDonald RB. Neurochemical alterations during age-related anorexia. Proceedings of the Society for Experimental Biology and Medicine 1999221 153-165.

2 McCue JB. The naturalness of dying. Journal of the American Medical Association 1995273 1039-1043.

3 Kalra SP \& Kalra PS. Is neuropeptide Y (NPY) a naturally occurring appetite transducer? Current Opinions in Endocrinology and Diabetes 19963 157-163. 